\title{
Propagation Characteristics of Cartesian Parabolic-Gaussian Beams
}

\author{
Lopez-Mago, D. ${ }^{a}$; Bandres, M. A. ${ }^{b}$; Gutiérrez-Vega, J. C. ${ }^{a}$ \\ ${ }^{a}$ Tecnológico de Monterrey, Monterrey, Mexico; \\ ${ }^{b}$ California Institute of Technology, California, USA
}

\begin{abstract}
We study the propagating and shaping characteristics of the novel one-dimensional Cartesian Parabolic-Gaussian beams. The transverse profile is described by the parabolic cylinder functions and are apodized by a Gaussian envelope. Their physical properties are studied in detail by finding the $2 n$-order intensity moments of the beam. Propagation through complex ABCD optical systems, normalization factor, beam width, the quality $M^{2}$ factor and its kurtosis parameter are derived. We discuss its behavior for different beam parameters and the relation between them. The Cartesian Parabolic-Gaussian beams carry finite power and form a biorthogonal set of solutions of the paraxial wave equation in Cartesian coordinates.
\end{abstract}

Keywords: Paraxial beams, Beam propagation, Intensity moments, Appell, Hypergeometric functions, Beam quality factor, $M^{2}$ factor, Kurtosis parameter

\section{INTRODUCTION}

Solutions of the paraxial wave equation (PWE) describe electromagnetic fields propagating in uniform and isotropic media. Since the behavior of laser propagation, resonators, waveguides, etc., can be described with the PWE, the study of different solutions of the PWE in different system of coordinates is a topic of interest because of the importance they have in these applications.

The purpose of this work is to fully analyze one novel and special family of solutions of the PWE in Cartesian coordinates. This family of solutions is a special case of the Cartesian beams and we have named such solutions the Cartesian Parabolic-Gaussian beams because its transverse intensity is expressed in terms of the parabolic cylinder functions and is modulated by a Gaussian envelope. The Cartesian Parabolic-Gaussian beams carry finite power and form a biorthogonal set of solutions of the PWE.

The physical properties are analyzed using the formalism presented in a recent paper by Bandres et. al. ${ }^{1}$ In that paper, the explicit expression for the higher-order moments and overlap of Cartesian beams is derived. Relevant beam parameters and invariants can be calculated from the moments of the beam, such as the beam power, normalization, the beam width, radius of curvature, the Kurtosis parameter and the beam quality factor. ${ }^{2}$ The kurtosis is a fourth-order parameter and describes the sharpness of flatness of the beam intensity distribution. The beam quality factor or $M^{2}$ is a second-order propagation invariant that has been accepted as an useful quantity for comparing and classifying the behavior of the beam with respect to an ideal Gaussian beam. The invariants such as the beam power and $M^{2}$ factor are important because they reflect physical properties that are inherent to the beam.

The physical realization of these beams can be performed by using experimental methods as the setup used by Durnin for generating Bessel beams ${ }^{3,4}$ or Fourier computer-generated holograms as used in the generation of Helmholtz-Gauss beams. ${ }^{5}$

We start our analysis giving a brief review of the Cartesian beams in section 2 to establish necessary formulas and notation. The expression for the overlap of the Cartesian beams and consequently the higher-order moments is revised in section 3. The shaping characteristics, propagation and properties of the Cartesian ParabolicGaussian beams are discussed in section 4 .

Laser Beam Shaping XI, edited by Andrew Forbes, Todd E. Lizotte, Proc. of SPIE Vol. 7789, 77890Q - @ 2010 SPIE · CCC code: 0277-786X/10/\$18 - doi: 10.1117/12.859283 


\section{CARTESIAN BEAMS}

Several closed-form solutions of the PWE in Cartesian coordinates have been studied over the years. The Cartesian beams were introduced as a new and very general beam solution. The complex amplitude of the Cartesian beams is described by the parabolic cylinder functions or the confluent hypergeometric functions, and the beams are characterized by three complex parameters. For special values of the beam parameters, the Cartesian beams reduce to many of the known solutions such as the standard, elegant, and generalized HermiteGaussian beams, ${ }^{6}$ the cosine-Gaussian and cosh-Gaussian beams, ${ }^{7}$ the Lorentz beams, ${ }^{8}$ the Airy beams ${ }^{9}$ and the fractional-order elegant Hermite-Gaussian beams. ${ }^{10}$

The Cartesian beams are described by the confluent hypergeometric function ${ }_{1} F_{1}(a, b ; x)$ as

$$
{ }_{t} U_{\beta}(x ; p, q)={ }_{t} \zeta_{\beta}\left(P x^{2}\right)^{(t-1 / 2) / 2}{ }_{1} F_{1}\left(\beta, t ; P x^{2}\right) \exp \left(\frac{i k x^{2}}{2 q}\right),
$$

which is characterized by three parameters $(p, q, \beta)$ that are complex in the most general case and the parity factor $t$ which takes the value

$$
t= \begin{cases}1 / 2, & \text { for even (e) beams, } \\ 3 / 2, & \text { for odd (o) beams }\end{cases}
$$

$k$ is the wavenumber and

$$
P=P(p, q) \equiv \frac{i k}{2}\left(\frac{1}{p}-\frac{1}{q}\right),
$$

where the complex parameters $(p, q)$ propagate from input plane $z_{0}$ to the output plane $z$ through an arbitrary real or complex ABCD optical system by the following transformation laws:

$$
p=\frac{A p_{0}+B}{C p_{0}+D}, \quad q=\frac{A q_{0}+B}{C q_{0}+D} .
$$

The scaling factor $\zeta_{t}$ comes from the propagation of the beam and becomes

$$
{ }_{t} \zeta_{\beta}=\frac{\left(A+B / q_{0}\right)^{\beta-(t+1 / 2) / 2}}{\left(A+B / p_{0}\right)^{\beta-(t-1 / 2) / 2}} .
$$

The fulfillment of the conditions $\operatorname{Im}\left(1 / p_{0}\right)>0$ and $\operatorname{Im}\left(1 / q_{0}\right)>0$ ensures the finiteness of the beam power across the whole transverse plane. The equation (1) in addition with the equations (3)-(5) characterize the cartesian beams through any paraxial ABCD system. The free-space propagation can be obtained by setting the values $[A, B ; C, D]=[1, z ; 0,1]$.

We remark that the Cartesian beams presented in Eq. (1) are a general solution of the one-dimensional PWE $\left(\partial_{x}^{2}+2 i k \partial_{z}\right) U(x, z)=0$, and if we want to construct a two-dimensional beam, it is obtained with the product of solutions in the $x$ and $y$ directions as $U(x, y ; z)={ }_{t_{x}} U_{\beta_{x}}\left(x ; p_{x}, q_{x}\right)_{t_{y}} U_{\beta_{y}}\left(y ; p_{y}, q_{y}\right)$.

The Cartesian beams reduce to families of optical beams with cartesian symmetry for special values of $\beta, p_{0}, q_{0}$. The possibility of choosing arbitrary values for the beam parameters have led to define novel and meaningful beam structures. ${ }^{1,11}$

\section{OVERLAP EXPRESSION FOR CARTESIAN BEAMS}

The complete analysis of the overlap and higher-order intensity moments of Cartesian beams was performed in a recent paper by Bandres et. al. ${ }^{1}$ In this section we present that formalism which will be used to calculate the shaping characteristics of the Cartesian Parabolic-Gaussian beams. The $2 \nu$-overlap of two Cartesian beams $U_{1}(x)={ }_{t_{1}} U_{\beta_{1}}\left(x ; q_{1}, p_{1}\right)$ and $U_{2}(x)={ }_{t_{2}} U_{\beta_{2}}\left(x ; q_{2}, p_{2}\right)$ with arbitrary parameters is given by the integral

$$
\sigma_{1,2}^{(2 \nu)}=\int_{-\infty}^{\infty} x^{2 \nu} U_{1}(x) U_{2}^{*}(x) \mathrm{d} x .
$$


We are going to restrict to the case where $t_{1}=t_{2}=t, U_{1}=U_{2}=U$ and $2 \nu=2 n=0,2,4, \ldots$, since we are interested in the higher-order intensity moments. With the conditions proposed, the integral reduces to

$$
\sigma^{(2 n)}=\int_{-\infty}^{\infty} x^{2 n}|U|^{2}(x) \mathrm{d} x .
$$

The evaluation of the expression (7) is given in the Eqs. (14)-(17) of Bandres et. al., ${ }^{1}$ it is:

$$
\sigma^{(2 n)}=\mathbf{b} \frac{\pi \Gamma(n+t)\left|\zeta_{t}\right|^{2}|P|^{t-1}}{S^{n+t}} F_{2}\left(n+t ; \beta, \beta^{*} ; t, t ; \frac{P}{S}, \frac{P^{*}}{S}\right),
$$

where

$$
\mathbf{b} \equiv \frac{1}{2 \pi}\left[1+(-1)^{2 n}\right]\left|P_{0}\right|^{1 / 2},
$$

and $S \equiv k \operatorname{Im}(1 / q)$ is a positive real quantity. Now, it is straightforward to calculate the moments of the Cartesian beam. The beam power corresponds to the zeroth-order intensity moment. It is calculated by setting $2 n=0$ in Eq. (8). The identity (9.182.3) of Gradshteyn ${ }^{12}$ reduces $\sigma^{(0)}$ in terms of the Gauss hypergeometric function $F={ }_{2} F_{1}(a, b ; c ; x)$ as follows:

$$
\sigma^{(0)}=\left|\frac{\Gamma(t) \bar{a}}{\bar{c}^{2 \beta} \bar{b}^{1 / 2-t}}\right| F\left(\beta, \beta^{*} ; t ; \bar{w}\right)
$$

where the quantities

$$
\begin{aligned}
\bar{a} & \equiv\left|\frac{1}{\left(A+B / q_{0}\right) \sqrt{S}}\right|=\frac{1}{\sqrt{S_{0}}}, \\
\bar{b} & \equiv\left|\frac{\left(A+B / p_{0}\right)}{A+B / q_{0}} \frac{P}{S}\right|=\left|\frac{P_{0}}{S_{0}}\right|, \\
\bar{c} & \equiv\left(1-\frac{P}{S}\right) \frac{A+B / p_{0}}{A+B / q_{0}}=1-\frac{P_{0}}{S_{0}} \\
\bar{w} & \equiv\left|\frac{P}{S-P}\right|^{2}=\left|\frac{P_{0}}{S_{0}-P_{0}}\right|^{2},
\end{aligned}
$$

remain invariant under propagation through paraxial systems characterized by unimodular ABCD matrices $(A D-B C=1)$ with real elements. The $2 n$-order moment of the Cartesian beams can be evaluated using the recursion formula

$$
\sigma^{(2 n)}=\left(-\frac{\mathrm{d}}{\mathrm{d} S}\right)^{n} \sigma^{(0)}, \quad n=1,2,3, \ldots,
$$

which is valid for even and odd parities. Using the recursive relation can be obtained the following expressions for the second-order intensity moments of the Cartesian beams.

Beams with even parity $(t=1 / 2)$

$$
{ }_{e} \sigma_{\beta}^{(2)}={ }_{e} G_{\beta e} \sigma_{\beta}^{(0)}+{ }_{e} H_{\beta o} \sigma_{\beta+1}^{(0)},
$$

where $e_{e} \sigma_{\beta}^{(0)}$ and ${ }_{o} \sigma_{\beta+1}^{(0)}$ are the powers of the beams ${ }_{e} U_{\beta}$ and ${ }_{o} U_{\beta+1}$, respectively, and the factors ${ }_{e} G_{\beta}$ and ${ }_{e} H_{\beta}$ depend on the beam parameters on the beam parameters upon

$$
\begin{aligned}
{ }_{e} G_{\beta} & \equiv \operatorname{Re}\left[\frac{2 \beta}{S}\left(\frac{P}{S-P}\right)+\frac{1}{2 S}\right], \\
{ }_{e} H_{\beta} & \equiv \operatorname{Re}\left[8|\beta|^{2} \frac{|P|}{S(S-P)}\left|\frac{A+B / p_{0}}{A+B / q_{0}}\right|\right] .
\end{aligned}
$$


Beams with odd parity $(t=3 / 2)$

$$
{ }_{o} \sigma_{\beta}^{2}={ }_{o} G_{\beta o} \sigma_{\beta}^{(0)}+{ }_{o} H_{\beta e} \sigma_{\beta+1}^{(0)}+{ }_{0} E_{\beta o} \sigma_{\beta+1}^{(0)},
$$

where ${ }_{o} \sigma_{\beta}^{(0)},{ }_{e} \sigma_{\beta+1}^{(0)}$ and ${ }_{o} \sigma_{\beta+1}^{(0)}$ are the powers of the beams ${ }_{o} U_{\beta},{ }_{e} U_{\beta+1}$, and ${ }_{o} U_{\beta+1}$ respectively, and the factors ${ }_{o} G_{\beta},{ }_{o} H_{\beta}$ and ${ }_{o} E_{\beta}$ depend on the beam parameters as

$$
\begin{aligned}
{ }_{o} G_{\beta} & \equiv \operatorname{Re}\left[\frac{3}{2 S}+\frac{2 \beta P}{S^{2} \bar{c}}\left(\frac{A+B / p_{0}}{A+B / q_{0}}\right)\right], \\
{ }_{o} H_{\beta} & \equiv \operatorname{Re}\left[-\left|\frac{\beta}{1 / 2-\beta}\right|^{2} \frac{\left|\bar{c}^{2}\right|}{S-P} \frac{(\bar{w}-1) \bar{b}}{2}\right], \\
{ }_{o} E_{\beta} & \equiv \operatorname{Re}\left[-\left|\frac{\beta}{1 / 2-\beta}\right|^{2} \frac{\left|\bar{c}^{2}\right|}{S-P}(1+4 \bar{w} \operatorname{Re} \beta)\right] .
\end{aligned}
$$

\subsection{General formulation for second-order moments}

The normalized second-order moments $\langle\hat{\rho}\rangle,\langle\hat{\tau}\rangle$ and $\langle\hat{\kappa}\rangle$ of the Cartesian beams are

- $\langle\hat{\rho}\rangle=\sigma^{(2)} / \sigma^{(0)}$ is the normalized second-order intensity moment of the beam.

- $\langle\hat{\tau}\rangle$ is related to the net momentum flowing in the transverse direction at the observation plane. The beam waist corresponds to the condition $\langle\hat{\tau}\rangle=0$. Positive (negative) values of $\langle\hat{\tau}\rangle$ mean that the beam is diverging (converging) as it propagates in the positive $\mathrm{z}$ direction.

- $\langle\hat{\kappa}\rangle=\hat{\sigma}^{(2)} / \sigma^{(0)}$ is the normalized second-order moment of the Fourier transform of the beam.

The explicit expressions for the values of $\langle\hat{\rho}\rangle_{0},\langle\hat{\tau}\rangle_{0}$ and $\langle\hat{\kappa}\rangle_{0}$ at the input plane in terms of the parameters of the Cartesian beams are

$$
\left[\begin{array}{c}
\langle\hat{\rho}\rangle_{0} \\
\langle\hat{\tau}\rangle_{0} \\
\langle\hat{\kappa}\rangle_{0}
\end{array}\right]=\operatorname{Re}\left\{\left[\begin{array}{ccc}
1 & 1 & 1 \\
1 / q_{0}^{*} & 1 / q_{0}^{*} & \left(1 / q_{0}+1 / p_{0}^{*}\right) / 2 \\
\left|1 / q_{0}\right|^{2} & 1 / q_{0}^{* 2} & 1 / q_{0} p_{0}^{*}
\end{array}\right]\left[\begin{array}{c}
J \\
K \\
L
\end{array}\right]\right\}
$$

where

$$
\begin{aligned}
J & \equiv \frac{t}{S_{0}} \\
K & \equiv \frac{2 \beta P_{0}}{S_{0}\left(S_{0}-P_{0}\right)}, \\
L & \equiv \frac{\beta}{t^{2}} \frac{P_{0} K^{*}}{\left|P_{0}\right|} \frac{{ }_{t} \sigma_{\beta+1}^{(0)}}{{ }_{t-1} \sigma_{\beta}^{(0)}} .
\end{aligned}
$$

The propagation laws of the normalized second-order moments $\langle\hat{\rho}\rangle,\langle\hat{\tau}\rangle$ and $\langle\hat{\kappa}\rangle$ through an ABCD system is given by

$$
\left[\begin{array}{c}
\langle\hat{\rho}\rangle \\
\langle\hat{\tau}\rangle \\
\langle\hat{\kappa}\rangle
\end{array}\right]=\left[\begin{array}{ccc}
A^{2} & 2 A B & B^{2} \\
A C & A D+B C & B D \\
C^{2} & 2 C D & D^{2}
\end{array}\right]\left[\begin{array}{c}
\langle\hat{\rho}\rangle_{0} \\
\langle\hat{\tau}\rangle_{0} \\
\langle\hat{\kappa}\rangle_{0}
\end{array}\right]
$$

Equation (24) permits the evaluation of the propagation of the second-order moments of a Cartesian beam through a paraxial ABCD system once their initial values are known. 


\section{CARTESIAN PARABOLIC-GAUSSIAN BEAMS}

The Cartesian beams offers the possibility of finding new and interesting beam structures. A novel family of solutions is given by setting the parameters of the Cartesian beams at plane $z=0$ as

$$
p_{0}=\frac{z_{R}}{h+i}, \quad q_{0}=-p_{0}^{*}, \quad \beta=\frac{t}{2}-\frac{i \alpha}{2},
$$

where $h \in \mathrm{R}$ and $z_{R}=k w_{0}^{2} / 2$ is the Rayleigh distance and $w_{0}$ is the waist of the Gaussian envelope. Putting $\left\{p_{0}, q_{0}, \beta\right\}$ into Eq. (1) we obtain

$$
{ }_{t} \Theta_{\alpha}(x ; z=0)=\frac{1}{2^{t / 2-1 / 4}} \exp \left(-\frac{x^{2}}{w_{0}^{2}}\right){ }_{t} \mathrm{P}_{\alpha}\left(\frac{2 \sqrt{h}}{w_{0}} x\right),
$$

where $t$ is the parity parameter which is $t=1 / 2$ for even beams and $t=3 / 2$ for odd beams, $\alpha \in \mathbb{R}$ is the order of the parabolic cylinder function. The parabolic cylinder function is expressed in terms of the confluent hypergeometric function ${ }_{1} F_{1}(a ; b ; x)$ as ${ }^{12}$

$$
{ }_{t} \mathrm{P}_{\alpha}(x)=\left(e^{i \pi / 4} x\right)^{t-1 / 2} \exp \left(-\frac{i x^{2}}{4}\right){ }_{1} F_{1}\left(\frac{t}{2}-\frac{i \alpha}{2} ; t ; \frac{i x^{2}}{2}\right),
$$

where is evident the influence of the parity factor $t$ in the parabolic cylinder function. The set of solutions given in Eq. (26) are exact solutions of the PWE in Cartesian coordinates. We have named this new family the Cartesian Parabolic-Gaussian beams because its transverse intensity is modulated by the parabolic cylinder function and is modulated by a Gaussian envelope. They were presented by Bandres and Gutiérrez-Vega ${ }^{11}$ in Eq. (16) as a special case of the Cartesian beams but its shaping characteristics have not been studied until now.

The free-space propagation is obtained by setting the matrix elements of the $\mathrm{ABCD}$ system as $[A, B ; C, D]=$ $[1, z ; 0,1]$ in Eq.(4), we get

$$
{ }_{t} \Theta_{\alpha}(x, z)={ }_{t} \zeta_{\alpha} \exp \left[\frac{i k}{4}\left(\frac{1}{p}+\frac{1}{q}\right) x^{2}\right]{ }_{t} \mathrm{P}_{\alpha}\left(\sqrt{k\left(\frac{1}{p}-\frac{1}{q}\right)} x\right),
$$

where $\{p, q\}=\left\{p_{0}+z, q_{0}+z\right\}$ are the propagation parameters controlling the scale of the gaussian envelope and the scaling factor ${ }_{t} \zeta_{\alpha}$ arises from the propagation of the beam and is given by

$$
{ }_{t} \zeta_{\alpha}=2^{1 / 4-t / 2} \frac{\left(q / q_{0}\right)^{i \alpha / 2-1 / 4}}{\left(p / p_{0}\right)^{-i \alpha / 2+1 / 4}}
$$

at the initial plane ${ }_{t} \zeta_{\alpha}=2^{1 / 4-t / 2}$. Equation (28) represents an exact closed-form solution of the one-dimensional PWE in free space. At any given transverse $z$ plane the Cartesian Parabolic-Gaussian beams are characterized by two real parameters $\alpha, h$ and the parity factor $t$. Two-dimensional solutions of the PWE in Cartesian coordinates can be constructed with the product of one-dimensional solutions, that is $U(x, y)={ }_{t_{x}} \Theta_{\alpha_{x}}(x)_{t_{y}} \Theta_{\alpha_{y}}(y)$, where any combination of parameters is possible. The propagation through a real ABCD optical system can be realized by using Eq. (4).

Figure [1] shows the transverse intensity distribution at $z=0$ ant at $z=2 z_{R}$ as well as the propagation plane of two-dimensional Cartesian Parabolic-Gaussian beams for special values of its parameters $\alpha, h$ and parity $t$. The Cartesian Parabolic-Gaussian beams have the property ${ }_{t} \Theta_{\alpha, h}(x)={ }_{t} \Theta_{-\alpha,-h}^{*}(x)$, hence we can analyze only the first two quadrants of the $(\alpha, h)$ plane.

\subsection{Normalization factor}

The zeroth-order moment $\sigma^{(0)}$ is defined as the integral $\sigma^{(0)}=\int_{-\infty}^{\infty} \Theta_{\alpha}(x)_{t} \Theta_{\alpha}^{*}(x) \mathrm{d} x$ that is equivalent to the power of the beam. In order to find the power of the Cartesian Parabolic-Gaussian beams, we can substitute the parameters $(p, q)$ in the equations (10) and (11). The power is conserved through any unimodular ABCD 


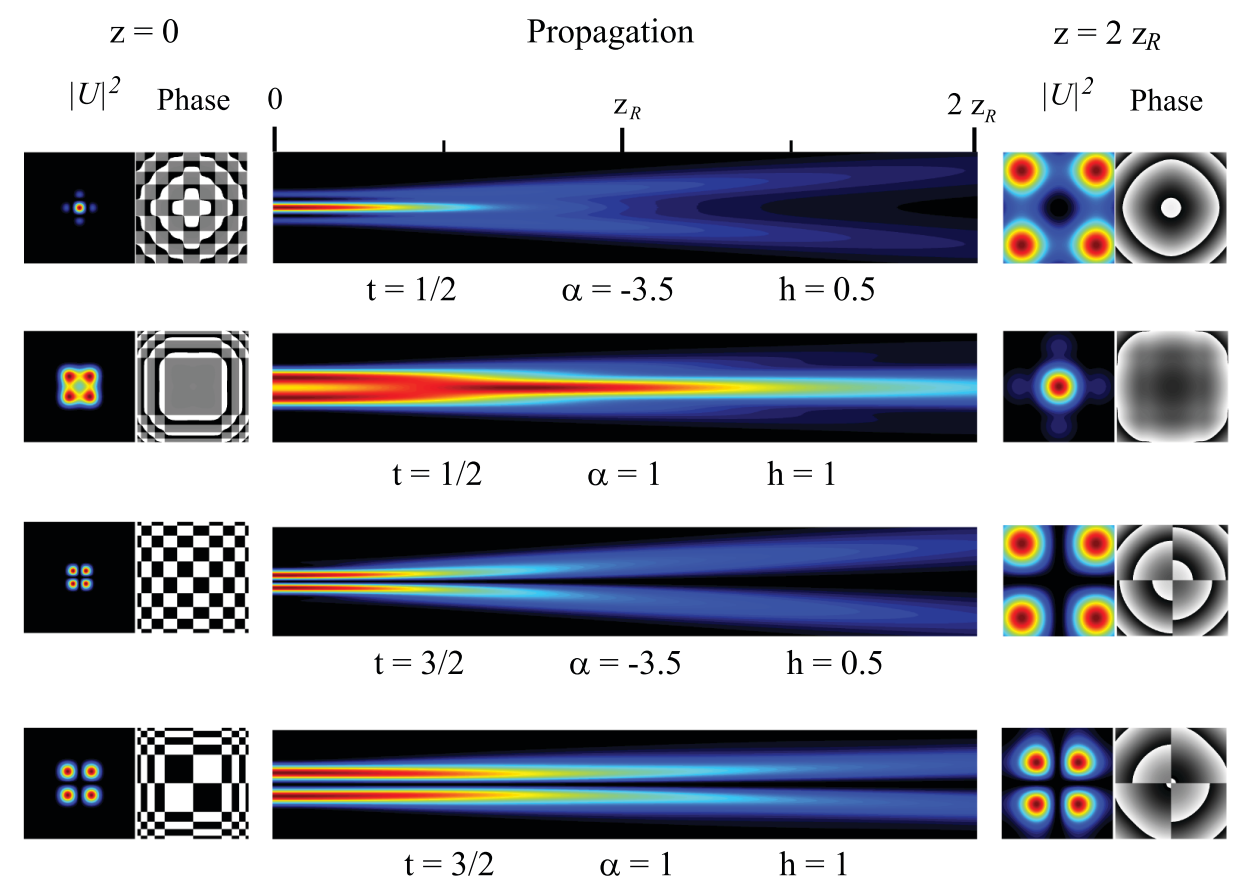

Figure 1. Intensity and phase patterns at plane $z=0$ and plane $z=2 z_{R}$ and along $\left(0 \leq z \leq 2 z_{R}\right)$, for even $(t=1 / 2)$ and odd $(t=3 / 2)$ two-dimensional Cartesian Parabolic-Gaussian beams $\Theta(x, y)={ }_{t_{1}} \Theta_{\alpha}\left(x ; p_{x}, q_{x}\right)_{t_{2}} \Theta_{\alpha}\left(y ; p_{y}, q_{y}\right)$. We have assumed $p_{0 x}=p_{0 y}$ and $q_{0 x}=q_{0 y}$.

optical system with real elements, meaning $A D-B C=1$. This property permits us to evaluate Eq. (10) with $p=p_{0}$ and $q=q_{0}$ to obtain

$$
\sigma^{(0)}=\frac{\Gamma(t) w_{0}}{\sqrt{2}}\left|\frac{h^{t-1 / 2}}{(1-i h)^{t-i \alpha}}\right| F\left(\frac{t}{2}-\frac{i \alpha}{2}, \frac{t}{2}+\frac{i \alpha}{2} ; t ; \frac{h^{2}}{1+h^{2}}\right) .
$$

Equation (30) is the first important result of this paper. It provides a simple expression to evaluate the beam power in terms of the initial beam width $w_{0}$, the parity factor $t$, the order $\alpha$ of the parabolic cylinder function and the parameter $h$. The normalization constant is written as $1 / \sqrt{\sigma^{(0)}}$.

\subsection{Beam width}

The beam width is related to the second-order moment by $w(z)=2 \sigma^{(2)}$. The second-order moment $\sigma^{(2)}=$ $\int_{-\infty}^{\infty} x^{2}{ }_{t} \Theta_{\alpha}(x)_{t} \Theta_{\alpha}^{*}(x) \mathrm{d} x$ for the Cartesian beams is expressed in Eqs. (13) and (16). We can find $\sigma^{(2)}$ of the Cartesian Parabolic-Gaussian beams by using the corresponding parameters $\{p, q\}$ and $\beta$. It is well known that for free-space propagation, the second-order intensity moment $\sigma^{(2)}$ has a parabolic function of the distance from the waist plane and it is related to the second-order moment at the far field $\tilde{\sigma}^{(2)}$ through the equation ${ }^{13}$

$$
\sigma^{(2)}=\sigma_{\text {waist }}^{(2)}+\left(\frac{\tilde{\sigma}^{(2)}}{k^{2}}\right)\left(z-z_{\text {waist }}\right)^{2},
$$

where the subindex waist means the plane of the beam waist. Using Eqs. (13) and $(16)$ with $[A, B ; C, D]=$ $[1, z ; 0,1]$ and rearranging terms we get

$$
\frac{\sigma^{(2)}}{\sigma^{(0)}}=\frac{w_{0}^{2} h}{2} \frac{u+\alpha}{h^{2}+1}+\frac{2 h}{k^{2} w_{0}^{2}}(u-\alpha) z^{2},
$$

in which $u$ is given by

$$
u=\frac{|t-i \alpha|^{2}}{2 t^{2}} \frac{{ }_{t} \sigma_{\alpha+i}^{(0)}}{t-1 \sigma_{\alpha}^{(0)}}+\frac{t}{h}
$$


where ${ }_{t} \sigma_{\alpha+i}^{(0)}$ and ${ }_{t-1} \sigma_{\alpha}^{(0)}$ correspond to the Eq. (30) but with $\alpha \rightarrow \alpha+i$ and $t \rightarrow t-1$ respectively. If we compare Eq. (31) with Eq. (32), we observe the value of $\sigma_{\text {waist }}^{(2)}$ and $\tilde{\sigma}^{(2)}$ is given by

$$
\begin{aligned}
\sigma_{\text {waist }}^{(2)} & =\frac{w_{0}^{2} h}{2} \frac{u+\alpha}{h^{2}+1}, \\
\tilde{\sigma}^{(2)} & =\frac{2 h}{w_{0}^{2}}(u-\alpha),
\end{aligned}
$$

and the position of the waist is always in the plane $z=0$ regardless the values of $\alpha$ and $h$.

\section{3 $M^{2}$ factor of Cartesian Parabolic-Gaussian beams}

Several beam parameters have been defined to measure the beam quality of a laser beam. The $M^{2}$ factor is a common parameter that relates the near-field beam size and the far-field beam spread of a laser beam and have gained such popularity that is currently used in data sheets of lasers. The $M^{2}$ factor is given in terms of the normalized second-order moments $\langle\hat{\rho}\rangle,\langle\hat{\tau}\rangle$ and $\langle\hat{\kappa}\rangle$ according to

$$
M^{2}=k \sqrt{\langle\hat{\rho}\rangle\langle\hat{\tau}\rangle-\langle\hat{\kappa}\rangle^{2}} .
$$

At the plane of the waist, $\langle\hat{\tau}\rangle=0$, and the $M^{2}$ factor reduces to

$$
M^{2}=k \sqrt{\langle\hat{\rho}\rangle\langle\hat{\tau}\rangle}=\sqrt{\frac{\sigma_{\text {waist }}^{(2)} \tilde{\sigma}^{(2)}}{\sigma^{(0)} \tilde{\sigma}^{(0)}}} .
$$

where $\sigma_{\text {waist }}^{(2)}$ and $\tilde{\sigma}^{(2)}$ are the second-order moment of the intensity and power spectrum of the beam, given in Eqs. (34) and (35), respectively. Because the plane of the waist for the Cartesian Parabolic-Gaussian beams is located at $z=0$, we can find the analytic expression for the $M^{2}$ factor by substituting Eqs. (34) and (35) into Eq. (37) to obtain

$$
M^{2}=\sqrt{\frac{u^{2}-\alpha^{2}}{1+h^{-2}}},
$$

where $u$ is given by Eq. (33). Figure [2] shows the numeric evaluation of the $M^{2}$ factor as a function of $\alpha$ and $h$ for even and odd beams. In both cases we can observe that the $M^{2}$ factor is an increasing function with respect to $h$ and $\alpha$. It is symmetric at the planes $h=0$ and $\alpha=0$. For even cases, the Cartesian Parabolic-Gaussian beams reduce to the one-dimensional Gaussian beam at plane $h=0$. It can be seen that regardless the value of $\alpha$, the $M^{2}$ remains constant at $1 / 2$, which agrees with the $M^{2}$ factor of the Gaussian beams. For odd beams, the minimum value $3 / 2$ is given at plane $h=0$.

\subsection{Kurtosis Parameter}

The kurtosis parameter describes the transverse intensity distribution of the beam. It determines the sharpness or flatness of the beam at a given longitudinal distant $z$. It is defined as ${ }^{14}$

$$
K=\frac{\sigma^{(4)} / \sigma^{(0)}}{\left(\sigma^{(2)} / \sigma^{(0)}\right)^{2}}=\frac{\sigma^{(0)} \sigma^{(4)}}{\left(\sigma^{(2)}\right)^{2}},
$$

where $\sigma^{(4)}$ corresponds to the fourth-order intensity moment, which can be calculated by using the recursive relation in Eq. (12), meaning $\sigma^{(4)}=\left(-\frac{\mathrm{d}}{\mathrm{d} S}\right)^{2} \sigma^{(0)}$. At a given $z$ plane, the beam profile is classified as leptokurtic (sharper profile), mesokurtic, or platykurtic (flatter profiles) depending on $K$ being larger, equal or less than 3, which is the kurtosis value of the one-dimensional Gaussian beam. ${ }^{14}$ Numerical evaluation of Eq. (39) is shown in Figure [3] for even and odd beams.

\section{CONCLUSIONS}

We have studied in detailed the physical properties of the novel Cartesian Parabolic-Gaussian beams. Important propagation factors were obtained through the higher-order intensity moments of the beam. Analytical expressions for the normalization factor, beam width, beam propagation factor $M^{2}$ and kurtosis parameter $K$ were obtained and numerical calculations performed for different values of the parameters. 

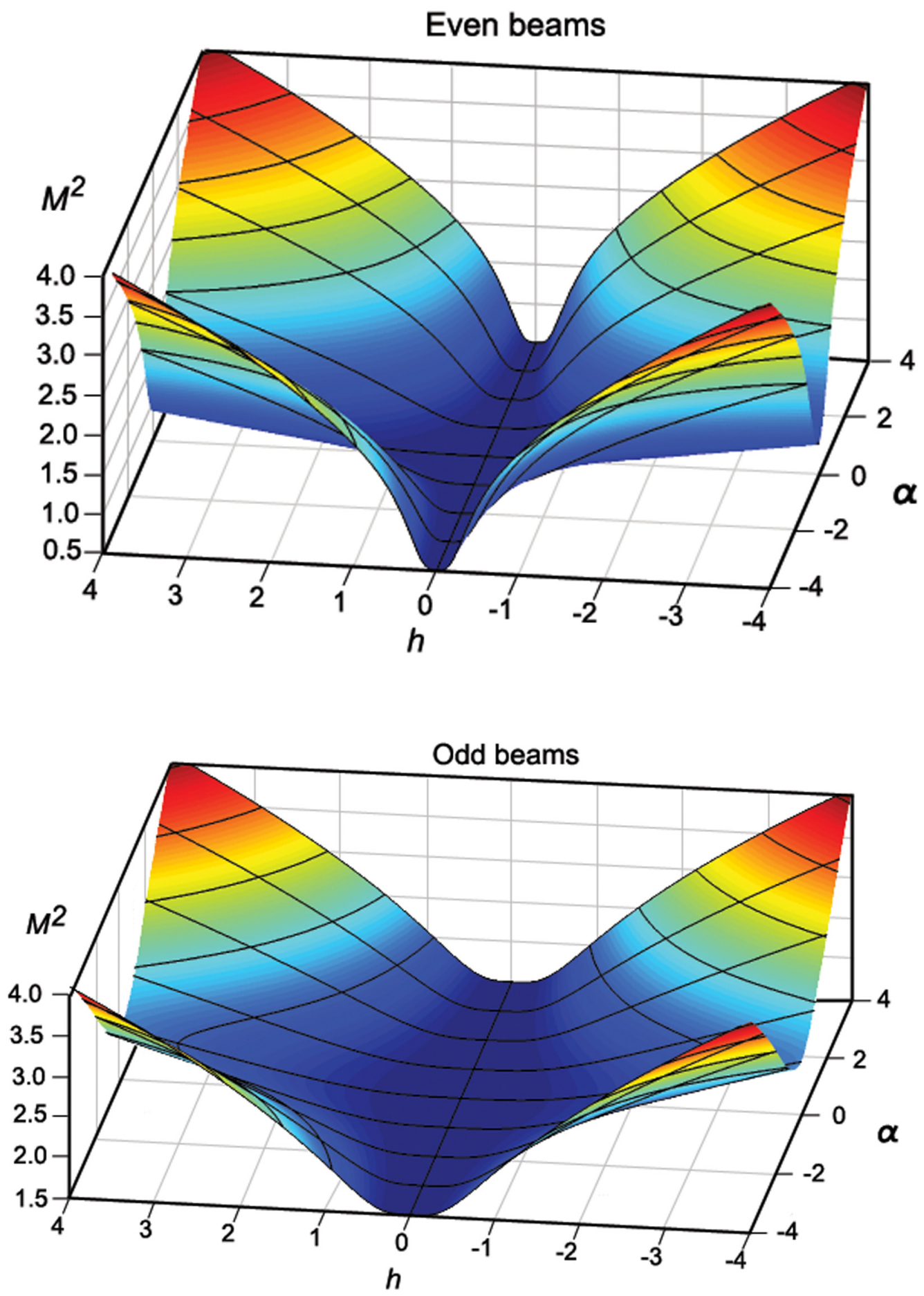

Figure 2. $M^{2}$ factor for even and odd Cartesian Parabolic-Gaussian beams. In both cases the $M^{2}$ factor is an increasing function of $h$ and $\alpha$, except for the case $h=0$ in which $M^{2}=1 / 2$ and $M^{2}=3 / 2$ for even and odd cases, respectively, and regarding the value of $\alpha$. For even Cartesian Parabolic-Gaussian beams, the $h=0$ case reduces to the one-dimensional Gaussian beam, which indeed have a $M^{2}=1 / 2$. 

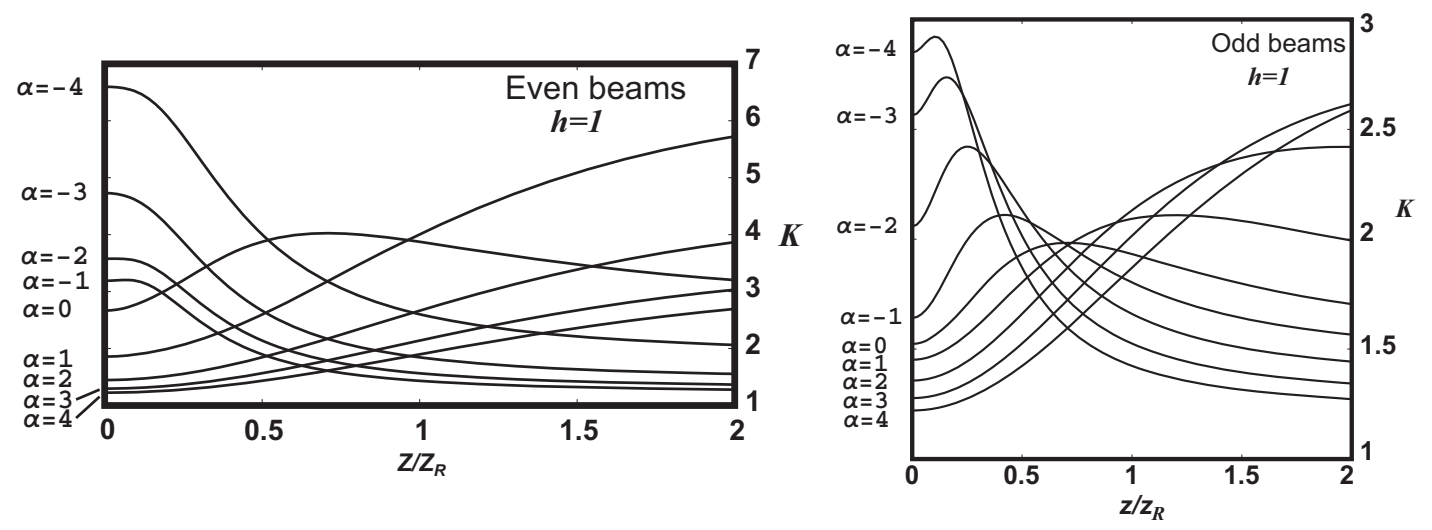

Figure 3. Kurtosis parameter for even and odd Cartesian Parabolic-Gaussian beams. We evaluate the kurtosis parameter $K$ for different values of the parameter $\alpha$ with $h=1$ and along $\left(0 \leq z \leq 2 z_{R}\right)$.

\section{ACKNOWLEDGMENTS}

We acknowledge support from Consejo Nacional de Ciencia y Tecnología (grant 82407) and from the Tecnológico de Monterrey (grant CAT-141).

\section{REFERENCES}

[1] Bandres, M. A., Lopez-Mago, D., and Gutiérrez-Vega, J. C., "Higher-order moments and overlaps of Cartesian beams," J. Opt. 12, 065702 (2010).

[2] Siegman, A. E., "New developments in laser resonators," Optical Resonators 1224(1), 2-14, SPIE (1990).

[3] Durnin, J., "Exact solutions for nondiffracting beams. I. The scalar theory," J. Opt. Soc. Am. A 4, 651-654 (1987).

[4] Durnin, J., Miceli, J. J., and Eberly, J. H., "Diffraction-free beams," Phys. Rev. Lett. 58, 1499-1501 (Apr 1987).

[5] López-Mariscal, C., Bandres, M. A., and Gutiérrez-Vega, J. C., "Observation of the experimental propagation properties of Helmholtz-Gauss beams," Opt. Eng. 45(6), 068001 (2006).

[6] Siegman, A. E., [Lasers], University Science Books (1986).

[7] Gutiérrez-Vega, J. C. and Bandres, M. A., "Helmholtz-Gauss waves," J. Opt. Soc. Am. A 22, 289-98 (2010).

[8] Gawhary, O. E. and Severini, S., "Lorentz beams and symmetry properties in paraxial optics," J. Opt. A: Pure Appl. Opt. 8, 409-14 (2006).

[9] Gutiérrez-Vega, J. C. and Bandres, M. A., "Airy-Gauss beams and their transformation by paraxial optical systems," Opt. Express 15, 16719-28 (2007).

[10] Gutiérrez-Vega, J. C., "Fractionalization of optical beams: I. Planar analysis," Opt. Lett. 32, 1521-1523 (2007).

[11] Bandres, M. A. and Gutiérrez-Vega, J. C., "Cartesian beams," Opt. Lett. 32(23), 3459-3461 (2007).

[12] Gradshteyn, I. S. and Ryzhik, I. M., [Table of Integrals, Series and Products], Academic (2007).

[13] Pierre-André, B., Ives, C., and Claude, P., "Beam propagation factor of diffracted laser beams," Opt. Commun. 105, 233-242 (1994).

[14] Martínez-Herrero, R., Piquero, G., and Mejías, P. M., "On the propagation of the kurtosis parameter of general beams," Opt. Commun. 115, 225-32 (1995). 